\title{
USING THE MD CAD MODEL TO DEVELOP THE TIME-COST INTEGRATED SCHEDULE FOR CONSTRUCTION PROJECTS
}

\author{
Chung-Wei Feng \\ Associate Professor, Dept. of Civil Engineering \\ National Cheng Kung University \\ No. 1, Ta-Hsueh Rd., Tainan City \\ 70101, Taiwan, R.O.C. \\ cfeng@mail.ncku.edu.tw
}

\author{
Yi-Jao Chen \\ Lecturer, Dept. of Asset Science \\ Leader University \\ No. 188, Sec. 5, An-Chung Rd., Tainan City \\ 70946, Taiwan, R.O.C. \\ yjchen@mail.leader.edu.tw
}

\begin{abstract}
This paper presents a work-item-based scheduling system that employs MD CAD model, Object Sequencing Matrix (OSM), and Genetic Algorithms (GAs) to generate the time-cost integrated schedule for construction projects. Consisting of MD CAD objects, the MD CAD model ensures the consistency and efficiency of information processing and integration in project planning. In the development of MD CAD model-based scheduling system, the Object Sequencing Matrix (OSM) is adopted to formulate a construction sequence of building components based on the physical relationships between MD CAD objects. Genetic algorithms (GAs) are then applied to optimize the sequence of building components and the assignment of crews. As a result, a feasible time-cost integrated project schedule is developed. In computer implementation, the MD CAD model-based Project Scheduling System (MD-PSS) is presented to verify the feasibility of the proposed approach.
\end{abstract}

\section{KEYWORDS}

MD CAD model; Construction schedule; Genetic Algorithms (GAs)

\section{INTRODUCTION}

Project planning is among the most critical factors to the success of a construction project. The planning process requires the transformation of data into decisions and actions. Information required for the execution of a project needs to be extracted from design and construction data. This information is then processed to formulate project knowledge necessary for making appropriate decisions and actions and the successful execution of the project. With this knowledge, a project plan is created as the overall schedule effectively manage construction project in terms of time and cost.

In construction industry, planners today commonly use the activity-based critical path method (CPM) to schedule the construction process. However, the current CPM scheduling framework for project planning does not provide enough information pertaining the spatial nature, resource, workspace, regulation, and etc. Planners need to study the $2 \mathrm{D}$ design drawings and contract documents, and investigate the site in order to further develop other construction plans. In practice, these construction 
plans are generally created independently by different individuals. This respective approach becomes a heavy burden to the project team due to the tremendous amount of information that must be manually integrated to develop a comprehensive plan [1]. For instance, it is still very difficult to integrate cost and schedule information for construction projects [2]. Correspondingly, it is hard to take account of both time and cost impact during the decision-making process. Some planners use the CPM method to integrate the product with the process, but this may lead to very detailed CPM schedules that are often difficult to use and to update during the construction process and lose their value as an instrument to plan and control work-flow [3].

Various research efforts have attempted to develop innovative ways to fully or partially automate the information processing and decision making during the planning processes. A promising approach that supports the planners by relating building components from a 3D CAD model to construction activities from a project schedule in one graphical environment is 4D CAD. Planners can simulate the construction process and analyze what-if scenarios in the $4 \mathrm{D} \mathrm{CAD}$ models. Planning supported by visual analyses of $4 \mathrm{D}$ CAD models is considered more useful and better than traditional planning [4]. However, 4D CAD models do not provide enough knowledge about the construction process itself [5]. The 4D CAD models are also difficult to update to reflect the construction progress and the most updated schedule.

This paper presents a work-item-based scheduling system that employs MD (multi-dimensional) CAD model, Object Sequencing Matrix (OSM), and Genetic Algorithms (GAs) to develop the time-cost integrated schedule for construction projects. This paper starts with analyzing the discrete processes undertaken within building construction in order to present the information of construction activities and specifications in the project model. These essential characteristics of the work items and building components are defined in the property sets of the proposed MD CAD objects [6]. These graphic objects are then utilized to create the MD CAD model, which will be further exported as a project database. Since the MD CAD objects are created based on work items, which are usually coded by certain standard format, the resources (e.g. labors, equipments, and materials) required for installation of building components can be easily retrieved. Moreover, the unit cost of a work item can be estimated accurately based on a defined crew (contains labors, equipments, and materials) that performs the tasks at an assigned production rate. An activity-based modeling approach is employed in this research to transform the project data into project planning information. In the development of MD CAD model-based scheduling system, the Object Sequencing Matrix (OSM) is adopted to formulate the construction sequence of building components based on the physical relationships between MD CAD objects. Genetic algorithms (GAs) are then applied to optimize the sequence of building components and the assignment of crews. As a result, a feasible time-cost integrated project schedule is developed.

This paper first discusses the activity-based CPM method and the model-based planning techniques in further detail. Next, the procedures of developing a MD CAD model and the proposed scheduling method are described. Finally, a computer implementation called MD CAD model-based Project Scheduling System (MD-PSS) is presented to verify the feasibility of the proposed approach.

\section{PROJECT SCHEDULING METHODS}

\subsection{Activity-based critical path method (CPM)}

In construction industry, the activity-based critical path method (CPM) has been widely applied for project scheduling. Planners first analyze the design and construction documents, and decompose a project into activities to develop a schedule network. However, the relationship between activities and activity's duration are decided according to experiences and the cognition of the planners. The outcome could be arbitrary since different planners could have different prospective of developing project schedule. In addition, the interdependency between activities and other impact factors, such as the relation between building components, the availability of resources, the site condition, the selection of construction method, the sufficiency of capitals, etc., which may influence the construction 
process, have been omitted. The current CPM method does not allow planners to explicitly describe the requirements and constraints of a construction project, which are very important for planning decisions. Therefore, planners can only determine the "role" (i.e., physical or technical impact) and "status" (i.e., flexibility of constrains) of activities in their minds, which is time-consuming and frequently prone to error [7].

Another difficultly with the current use of the CPM scheduling method for construction planning is related to the information processing of a project. Schedules generated form the CPM method are based on activities, while in construction process, the project is executed according to work items of the contracts and subcontracts. The work items contain the cost data of the project, but they are not connected to the activities of the project schedule. It is because each activity may include several work items and each work items may be included by several different activities with various amounts of works. Consequently, if the project consists of thousands of activities, it is infeasible for the project planner to connect the work items to the activities to develop a time-cost integrated project schedule.

\subsection{Model-based project planning}

Embarking on advancements in computer graphics, information systems, and artificial intelligence, various research efforts have attempted to automate the planning process by developing tools to manipulate and process project information, carry out the decision-making, and propose the required action. The development of 4D CAD model that enable graphic simulation and visualization of construction process has become the current trend among studies on model-based planning system. The 4D CAD model combines 3D CAD model with project schedule and represent the construction plan graphically. Various studies have acknowledged its capabilities on construction planning, construction management strategies, and site management [8-10].

4D CAD models have maximized the functionalities of $3 \mathrm{D}$ CAD models and help to increase the interactions between project participants. Besides, 4D CAD models may assist in analyzing the possible time-space conflicts, safety issues and site workspaces management, as well as in identifying and reducing construction-related problems in the pre-construction phase [4]. However, in project planning applications, 4D tools still have some obstacles to overcome [3,8]. The 3D CAD models used in most of today's 4D CAD models are building components. The information embedded in the building components which is sufficient for project designs is often insufficient to meet the requirements of project planning. Accordingly, the use of $4 \mathrm{D}$ CAD models still requires the planner manually relate building components to construction activities and visually test whether problems occur during the construction process. Therefore, in the development of model-based planning system, more construction knowledge and project information should be incorporated in the models to effectively assist planners in project planning.

\section{MD CAD MODEL}

\subsection{Building Information Modeling}

Current research in information modeling techniques is a good foundation for integrating design and construction information for construction planning and management. The Building Information Modeling (BIM), with its data-rich digital representation, is known as an innovative approach to cataloging the physical and functional characteristics of design and construction [11]. Currently, BIM has been recognized to be essential in AEC industry for its capabilities of managing, sharing and exchanging information among project participants throughout the project lifecycle. In the developments of BIM applications, the IFC (Industry Foundation Classes) data model [12] has been widely adopted. However, the current IFC is still incapable of providing sufficient information required by the construction activities [9]. Although the model schema of building product is now available, standards in construction management domain still need to be defined.

To use BIM effectively in construction projects, the information processing between different participants in the construction process has to be more efficient [13]. In addition, the relationships among the building components and activities are also crucial to an integrated BIM [14]. In order to present the 
information required for project planning in project models. This study starts with analyzing the discrete processes undertaken within building construction to define essential attributes of the work items and building components. The information of construction activities and specifications are generated through the suggested activity-based modeling approach, which can create the relationships between work items and building components in the proposed MultiDimensional (MD) CAD objects. The MD CAD model is intended to transform and integrate project information for the purpose of construction planning and management.

\subsection{Framework of MD CAD model system}

The proposed MD CAD model system will be developed as a computer system, which will focus on conducting project information and decision supports for project planning. The MD CAD model will be adopted as core project information repository, where initial project data from various design/contract documents will be retrieved and further transformed into the information required for the execution of the project. The diagram in Fig. 1 shows the framework of the MD CAD model system. According to this diagram, the multidisciplinary data generated by various project participants is collected in pre-construction phase. These data are then used to create the MD CAD objects in an IFC-based CAD system. The MD CAD model is composed of these MD CAD objects.

An MD CAD model is an extension of a BIM model, which incorporates multi-aspects of construction information required for project planning and management. The attributes of a MD CAD object are divided into geometric, containment hierarchy, and behavioral aspects. In geometric aspect, the attributes include the coordinates, measurements, and physical relationships of a building component. The measurements information will be utilized as a reliable source in quantity estimation for a project. The physical relationships between building components can be used to generate an optimal sequence of construction, this sequence will be further served as a basis on scheduling. In the containment hierarchy, the MD CAD objects are classified into physical, spatial, and logical objects. This containment hierarchy defines the model structure of the MD CAD model. In behavioral aspects, the construction contents are described by the work items in an appropriate level in the containment hierarchy. The work items employed in this research are classified and encoded according to a hierarchical standard code [15]. Based on the standard code of a work item, the information of the corresponding specification and resource items can be obtained.

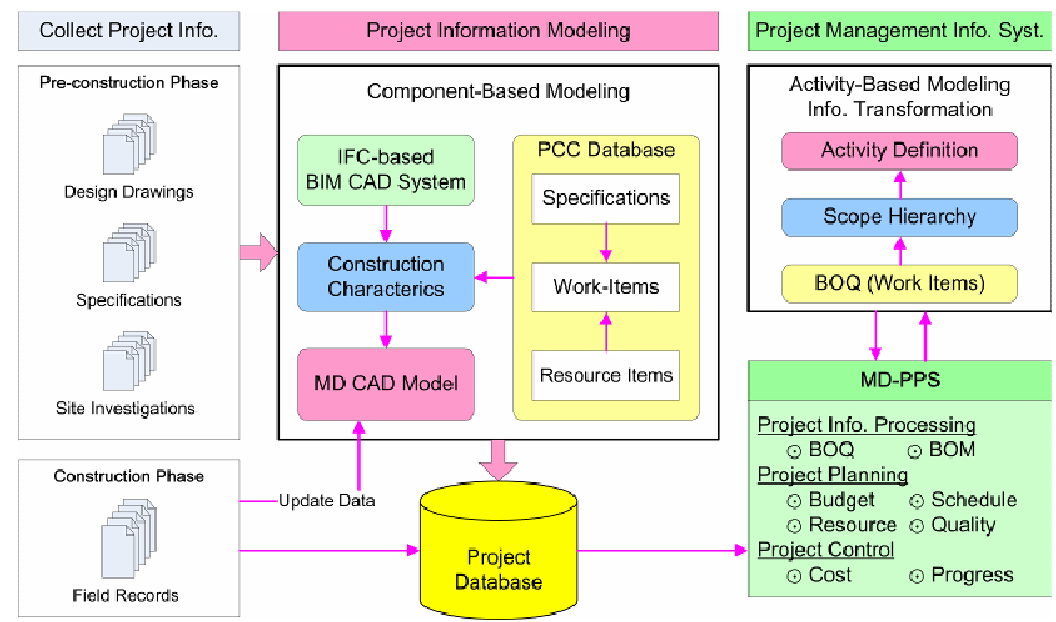

Figure 1. The framework of MD CAD model-based system. 
These essential attributes of the work items and building components are defined in the property sets of the MD CAD objects. After the creation of the MD CAD model, the information described in the MD CAD model will be exported to a project database. Thus, all project-required work can be identified on the basis of collected work items in the MD CAD model. In the model, even though the work items described in the MD objects can represent the construction contents, they are still incapable of generating information on activity items. This limitation arises because the relationships between work items and activity are not established. Moreover, since the componentbased modeling approach defines the components' structure in BIM model from the perspective of building design, the graphic objects used in BIM can only be used to show the ultimate appearance of the designed building. In order to transform the project data into the information for project planning, this research adopts an activity-based modeling approach as a mechanism of information transformation. In this transformation mechanism, a scope hierarchy structure is established to define the activity items in a consistent formation (Fig. 2). This organized manner will help integrating project information into a standard format for cost estimating, project scheduling and project control.

In the MD-PSS system, project information such as BOQ (Bill of Quantity), BOM (Bill of Materials), and resource requirements can be produced efficiently and accurately to support future budget and schedule planning. In addition, during the construction phase, the filed records are recorded in the MD-PSS to assist the project team for cost and schedule control. Based on the project performance evaluations, the project plans can be modified according to the performance indexes such as CPI (Cost Performance Index) and SPI (Schedule Performance Index).

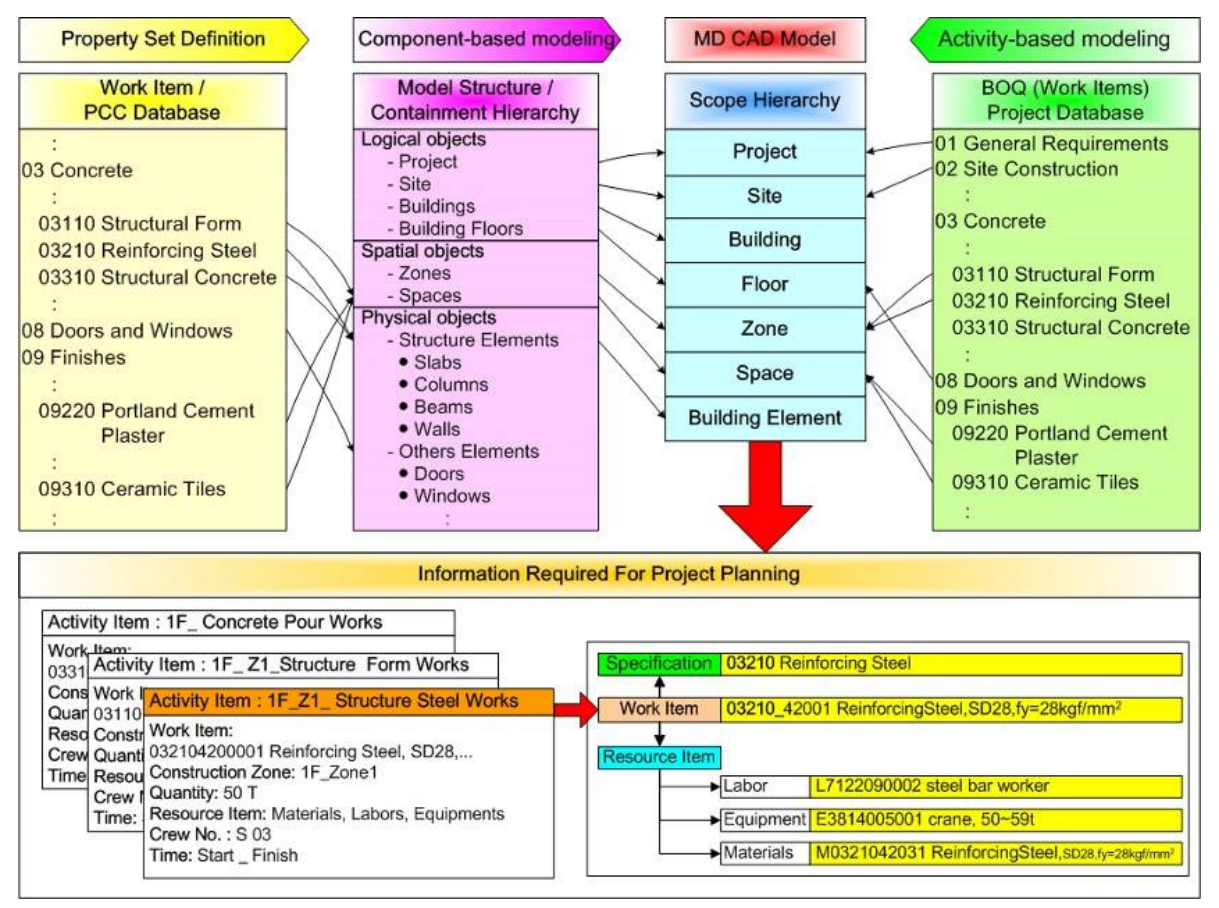

Figure 2. Activity-based modeling process. 


\section{MD CAD MODEL-BASED SCHEDULING SYSTEM}

In the development of the MD CAD model-based scheduling system, this research employs the Object Sequencing Matrix (OSM) and Genetic Algorithms (GAs) to develop a time-cost integrated schedule for construction projects. The OSM is adopted to represent the construction sequence of building components based on the physical relationships between MD CAD objects [16]. Then, the Genetic Algorithms (GAs) is applied to optimize the construction sequence and to assist in crew assignment, which takes into consideration the constraints on resource, workspace, and productivity.

For now, this research focuses on the scheduling of cast-in-place concrete engineering. The construction activities include the Structure Steelworks, Structure Formworks, and Concrete Pour Works.

\subsection{Construction Sequence of building components}

In the proposed project scheduling method, the physical relationship between building components, which is defined by the geometric attributes retrieved from the MD CAD objects, is utilized to formulate the construction sequence of the components and is presented in OSM. As shown in Fig. 3, the circled "S-D" indicates that object $\mathrm{C} 1$ (column) is "supported directly by" object B1 (beam), the circled "S-I" indicates that object W1 (wall) is "supported indirectly by" object B3 (beam), and the circled " $\mathrm{D}$ " indicates that object W3 (wall) "depends on" object C1 (column).

The sequence of objects in OSM is the construction order of building components. As shown in Fig. 3, the circled "S-I" in the upper-right zone indicates that object $\mathrm{C} 1$ is supported indirectly by object $\mathrm{B} 4$, i.e. object $\mathrm{B} 4$ must be installed before object $\mathrm{C} 1$. Therefore, object B4 should be scheduled before object $\mathrm{C} 1$. In other words, the object relationships in the upper-right zone of the matrix should be all moved to the lower-left zone, which is the rational sequence of the building components. Based on these precedence relationships between building components and regardless of the construction continuity, a number of possible construction sequences will be developed. In order to generate a more practical construction sequence for building components, the relationships in OSM conformed to the continuity condition will obtain a higher weight value in the sequencing algorithm (Fig. 3).

For now, this research only looks into the inflexible constraints (the relationships of supported directly by, supported indirectly by, and depend on) between components; the flexible constraints between components will be discussed in our future research.
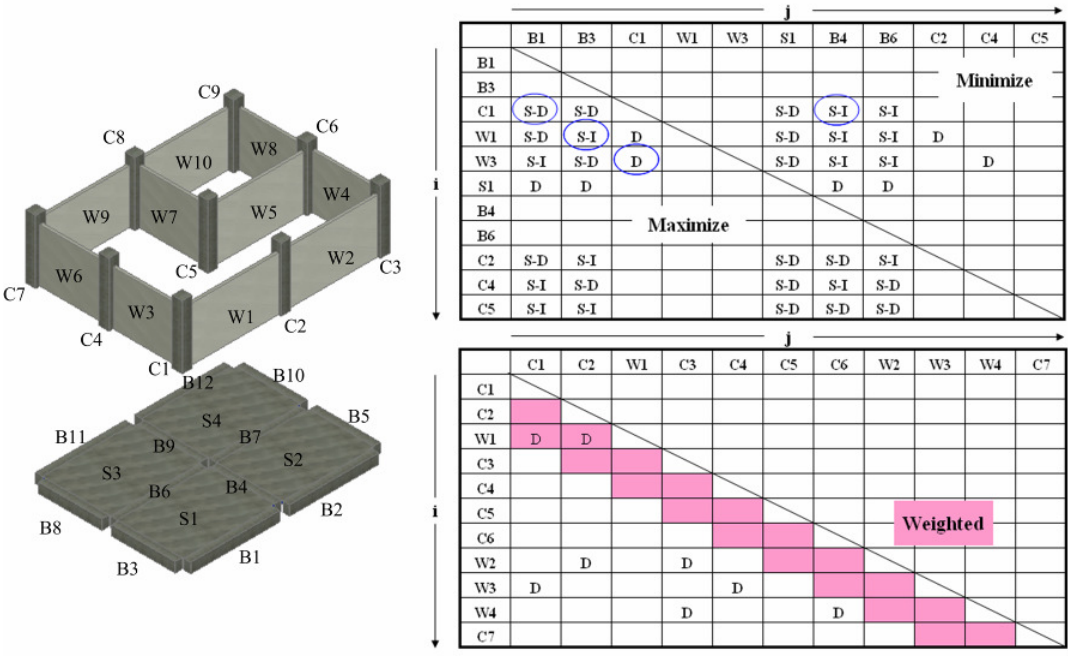

Figure 3. Object Sequencing Matrix (OSM). 
The Genetic Algorithms (GAs) are applied to search for an optimal construction sequence of the building components. The initial sequence of building components is randomly generated. The relationships between building components appeared in the OSM will be transformed into values. In this research, the value of relationships with inflexible constraint is set as 1 ; otherwise the value is set as 0 . In addition, the weighted value of relationships that conform to the continuity condition is set as 2 . As mentioned above, to obtain a rational construction sequence of building components, the relationships appear in the upper-right zone of the matrix should be moved to the lower-left zone, i.e. the sum of values in the upper-right zone should be minimized (the appropriate value $=0$ ). Hence, the fitness function is given as Eq. (1). While all the relationships are moved to the lower-left zone of the matrix, the sum of the weighted values should be maximized. The fitness function is given as Eq. (2):

$$
\begin{aligned}
& \text { Min. } f(m)=\left(\sum_{i=1}^{n-1} \sum_{j=i+1}^{n} \operatorname{OSM}(i, j)\right) \\
&, n=\text { the number of objects } \\
& \text { Max. } g(m)=2 \times\left(\sum_{j=1}^{n-D} \sum_{i=j+1}^{j+D} \operatorname{OSM}(i, j)+\sum_{j=n-(D-1) i=j+1}^{n-1} \sum_{i=1}^{n} \operatorname{OSM}(i, j)\right) \\
&+\left(\sum_{j=1}^{n-(D+1)} \sum_{i=j+(D+1)}^{n} \operatorname{OSM}(i, j)\right),
\end{aligned}
$$

$n=$ the number of objects, $D=$ the given continuity condition

Eq. (2).
The optimal construction sequence of building components has considered both the physical relationship of the components and the construction continuity. Therefore, this sequence can be further served as a basis for scheduling.

\subsection{Optimal crew assignment}

This section provides a detailed description of using GAs to obtain an optimal crew assignment of scheduling. Firstly, the chromosome structure and the decoding process of the genetic string are described with an example. Secondly, the fitness value that measures the survivability of the genetic string is explained. Finally, the evolutionary mechanisms adopted in this method are illustrated.

\section{Chromosome Structure}

In GAs, a chromosome string represents a set of decisions, namely chromosome combination, and infers a potential solution to the problem. The weekly crew assignments for project-required work items are presented in a chromosome string. As shown in Fig. 4 , in a workspace that allows a maximum of 10 crews, the genetic code (1) 820 means that the assignment of crews to Steel Works, Form Works, and Concrete Works in the first week is 8 crews, 2 crews, and none. The initial crew assignment are randomly generated and the strings are input into $\operatorname{Str}(m, n)$, while $m$ is the size of initial population (1 Pop_Size), and $n$ is the length of the string $(n=1 \sim N \times 4, N$ : the weeks of schedule). Moreover, the number of assigned crews is limited by the workspace and resource constraints. Confronting the constraints on workspace and resource, how to maximize the amount of work completed by the crews is crucial to the optimization of crew assignment.

\begin{tabular}{c|c|c|c|c|}
\begin{tabular}{c|c} 
Gene \\
(Weekly Crew Assignment)
\end{tabular} & (Week) & $\begin{array}{c}\text { Crews for } \\
\text { Steel Works }\end{array}$ & $\begin{array}{c}\text { Crews for } \\
\text { Form Works }\end{array}$ & $\begin{array}{c}\text { Crews for } \\
\text { Concrete Works }\end{array}$ \\
\cline { 2 - 5 }
\end{tabular}

\begin{tabular}{c|c|c|c|c|c|c|c|c|c|c|c|c|c|c|c|c|c|}
$\begin{array}{c}\text { Genetic } \\
\text { Code }\end{array}$ & $(1)$ & 8 & 2 & 0 & $(2)$ & 0 & 0 & 4 & $(3)$ & 3 & 0 & 7 & $\cdots \cdots$ & (N) & 4 & 1 & 2 \\
\hline
\end{tabular}

Figure 4. The chromosome structure. 


\section{Fitness Value}

Under workspace and resource constraints, crew assignment is optimized to provide the highest capacity utilization. Therefore, the fitness value of a crew assignment is defined as the higher the capacity utilization the better the fitness value of a crew assignment is. In crew assignment, the capacity and the scheduled amount of work should be calculated in order to evaluate capacity utilization. After a genetic string is generated, the capacity that could be provided by the assigned crews may be calculated according to Eq. (3).

$$
\begin{aligned}
& \left\{\begin{array}{l}
\text { Capacity_steel }(m)=\left(\text { pro_steel } \times \sum_{k=0}^{N-1} \operatorname{Str}(m, 4 k+2)\right) \times \text { days } \\
\text { Capacity_form }(m)=\left(\text { pro_form } \times \sum_{k=0}^{N-1} \operatorname{Str}(m, 4 k+3)\right) \times \text { days } \\
\text { Capacity_concrete }(m)=\left(\text { pro_concrete } \times \sum_{k=0}^{N-1} \operatorname{Str}(m, 4 k+4)\right) \times \text { days }
\end{array}\right. \\
& \begin{array}{l}
\text { Nethe weeks of schedule, } \\
\text { days }=\text { workdays, } \\
\text { pro_=production rate }
\end{array}
\end{aligned}
$$

Given the capacity, the scheduled amount of work of each work item can be calculated based on the installable building components that are sequenced in OSM.

In order to prevent the scheduled amount of work less than the required amount of each work item, a penalty function is given as Eq. (4). Hence, the fitness function is given as Eq. (5).

$$
\text { If Scheduled_ }(m)<\sum_{x=1}^{\text {num }} O S M_{-}(x),
$$

Then Scheduled_ $(m)=0$

Maximum Fitness Value $=\left(\frac{\text { Scheduled_steel }(\mathrm{m})}{\text { Capacity_steel }(\mathrm{m})}\right)$

$+\left(\frac{\text { Scheduled_form }(m)}{\text { Capacity_form }(m)}\right)+\left(\frac{\text { Scheduled_concrete }(m)}{\text { Capacity_concrete }(m)}\right)$

\section{Evolutionary Mechanism}

The evolutionary mechanism follows the procedures of selection, crossover, mutation, etc. There are two main selection mechanisms, namely elitist selection and roulette wheel selection. In elitist selection, the optimal solutions with highest fitness value are retained and duplicated to the new population. That is, this selected part will not participant in the crossover and mutation procedure. Its evolution from current generation to the next generation is safeguarded to ensure a better development. The remaining will be selected through roulette wheel mechanism. Those chromosomes with better fitness value have higher probability to be selected to the new population. Then, the uniform crossover is applied for chromosomes' crossover. After this procedure, several genes in the chromosome will undergo the process of mutation in order to ensure that the solution is in global optimum instead of local optimum.

After GAs' evolution and convergence procedures, the optimal construction sequence of building components and the optimal crew assignment are generated.

\section{COMPUTER IMPLEMENTATION}

This research applies the AutoCAD Architectural 2008 for the creation of the MD CAD model. In order to facilitate the process of creating MD CAD model, the prototype of the MD CAD objects will be systematically saved in a MD Object Database. The diagram in Figure 5 demonstrates how a MD CAD model is developed. The data collected and integrated through the MD CAD model will be exported to a database file, which will be further applied to the MD-PPS system. The major function of MD-PPS is to generate useful information for project planning and control.

There are six major sections in the MD-PPS system:

1. General Information: This section records the general information of a project, such as project name, project number, site address, construction date, and contract total cost.

2. BOQ and BOM: The quantity take-off is an important part in cost estimation and schedule planning. In this section, based on the project database imported from the MD CAD model, project information such as BOQ (Bill of Quantity), BOM (Bill of Materials) and resource requirements can be efficiently and accurately produced. The activity items can be identified on the basis of the project-required work items. Descriptions are created to describe the scope and 
material requirement for each activity item. The number and other detail information related to the listed activities will vary from job to job and will depend on the intended level of planning.

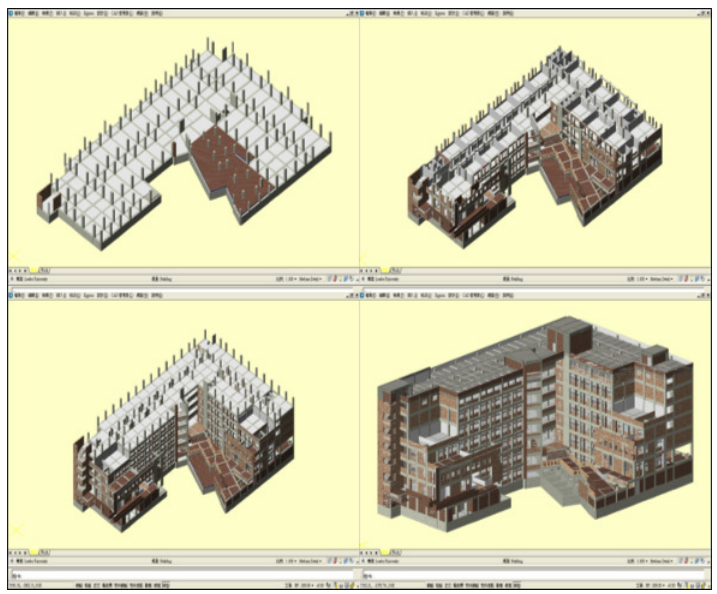

Figure 5. The development process of a MD CAD model

3. Scheduling Process (Figure 6): In the initial process of project's scheduling, certain parameters should be inputted by the planners, including the start-finish date of the project, the production rate of each work item, the resource and workspace constraints. The parameters of GAs include population size, mutation rate, crossover rate and number of generation. Next, an optimal construction sequence of building components should be established in OSM. According to the sequence, each component is provided with ID number and the quantity of each required work item is calculated automatically by MD-PSS. With the inputted parameters and the sequence, planners may formulate an optimal crew assignment (as shown in Figure 6). Also, the weekly crews assigned to each work item and the scheduled amount of each item will be provided in this section.

4. Construction Plan (Figure 7): In this section, the construction process is scheduled in a bar chart. In time-cost integration, the cash flow of the project is estimated based on the weekly scheduled amount of work and the accumulated cash flow is shown in the bar chart. Differed from the activity-based CPM scheduling method, this research employs a work-item-based scheduling method in order to formulate a timecost integrated project schedule. Furthermore, the work items employed in this research are classified and encoded according to a hierarchical standard code. Based on the standard code of a work item, the information of the corresponding specification and resource items can be obtained. In other words, MD-PSS is capable of accurately calculating the weekly requirement of the quantity of labor, equipment and material for the purpose of resource management and control.

5. Site Record: The system is capable of generating daily field records for tracking the construction work. The information includes the completion of work items, resource consumption and cost.

6. Project Performance (Figure 8): Effective planning requires continuous monitor so that the manager can make forecast and revise plans to maintain the proper course toward the objective. In this section, the data obtained from "Construction Plan" and "Site Record", such as scheduled completion, budgeted cost, actual completion, and actual cost, will be used in earned value analysis. The cost and schedule variances assist in evaluating and controlling project risk by measuring progress in monetary terms.

\section{CONCLUSIONS}

This research proposes a MD CAD model-based scheduling method to explicitly describe the requirements and constraints of a construction project, which are crucial to planning decisions. In a MD CAD model, since the MD CAD objects are created based on work items, which are coded by standard format, the resources and cost required for installation of building components can be easily retrieved. Furthermore, the application of the Genetic Algorithms (GAs) helps to optimize the construction sequence and to assist in crew assignment, which takes into consideration the constraints on resource, workspace, and productivity. The proposed MD-PPS system has demonstrated the efficiency and feasibility of the proposed approach in project planning and control. 


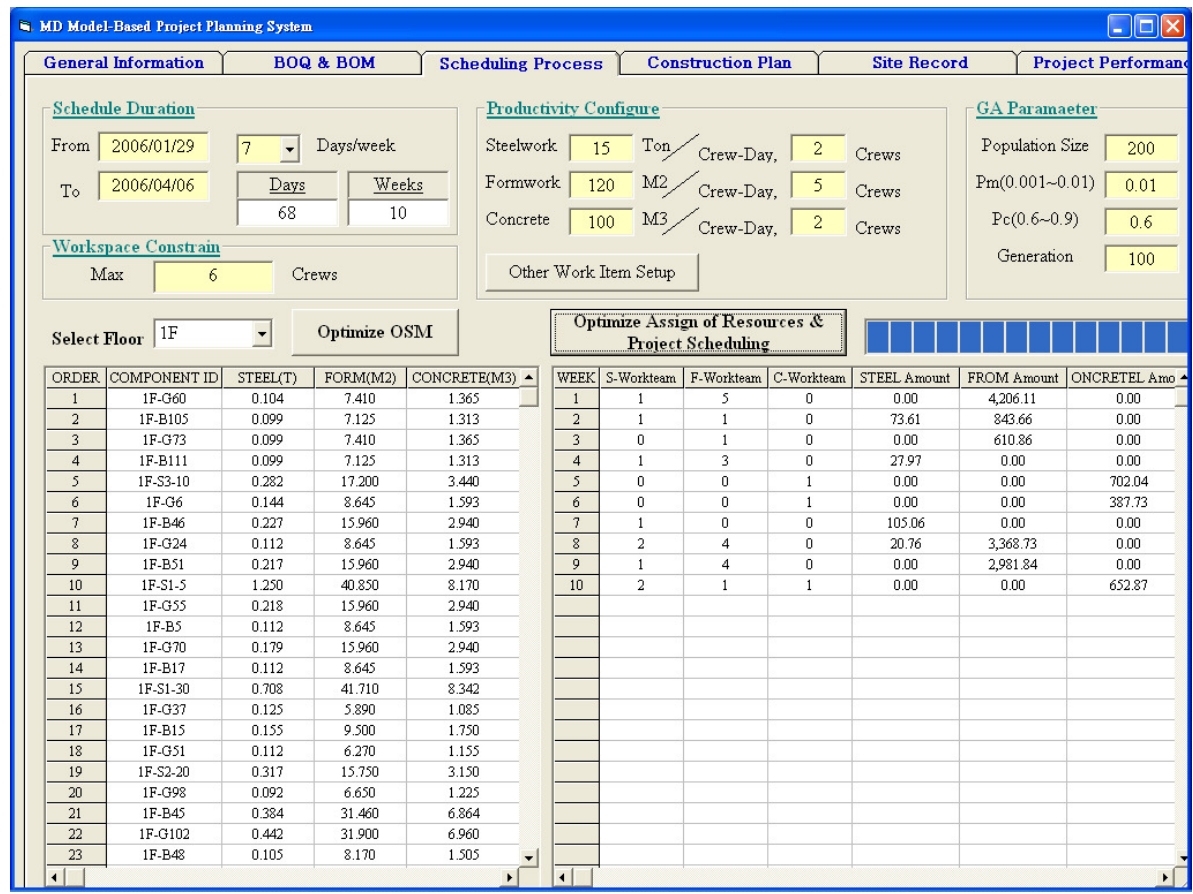

Figure 6. The "Scheduling Process" section in MD-PPS

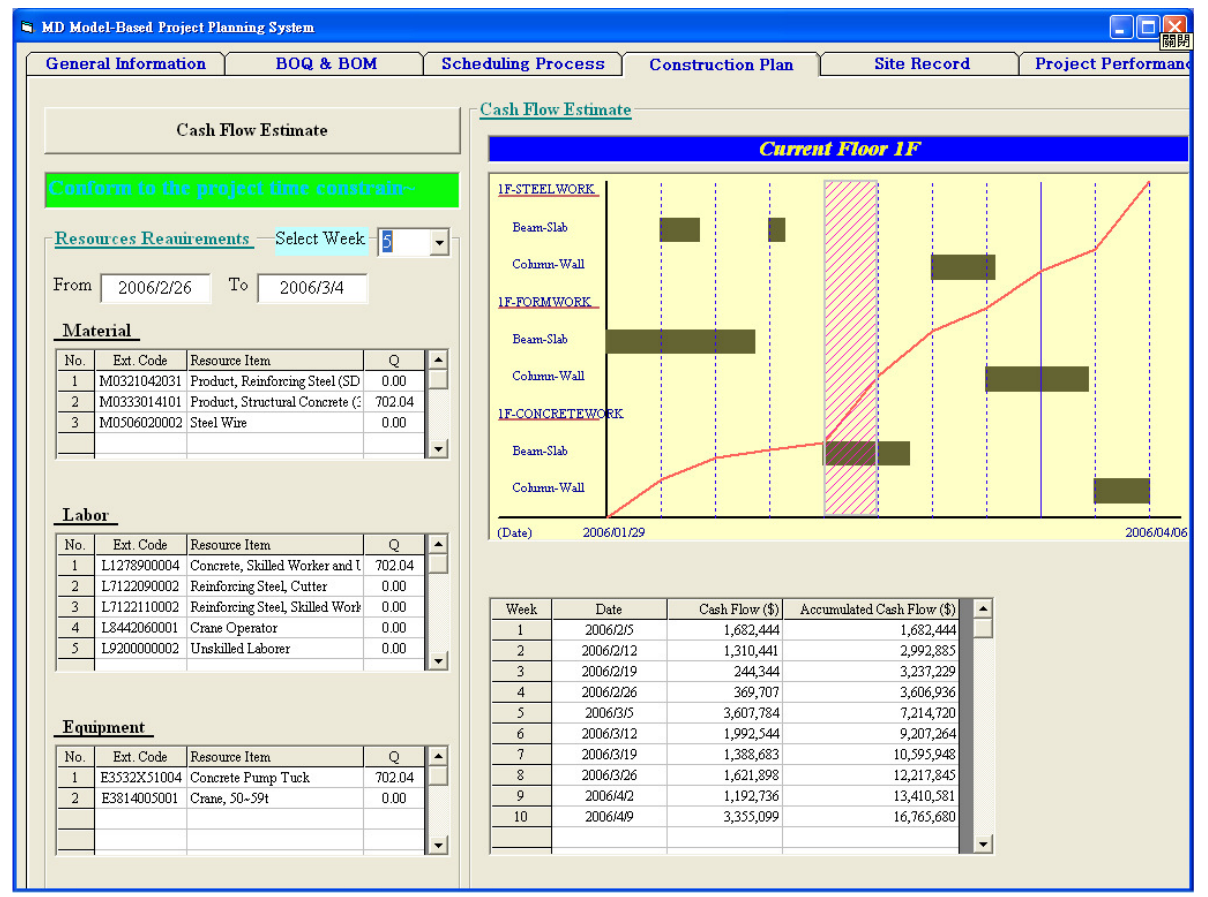

Figure 7. The "Construction Plan" section in MD-PPS 


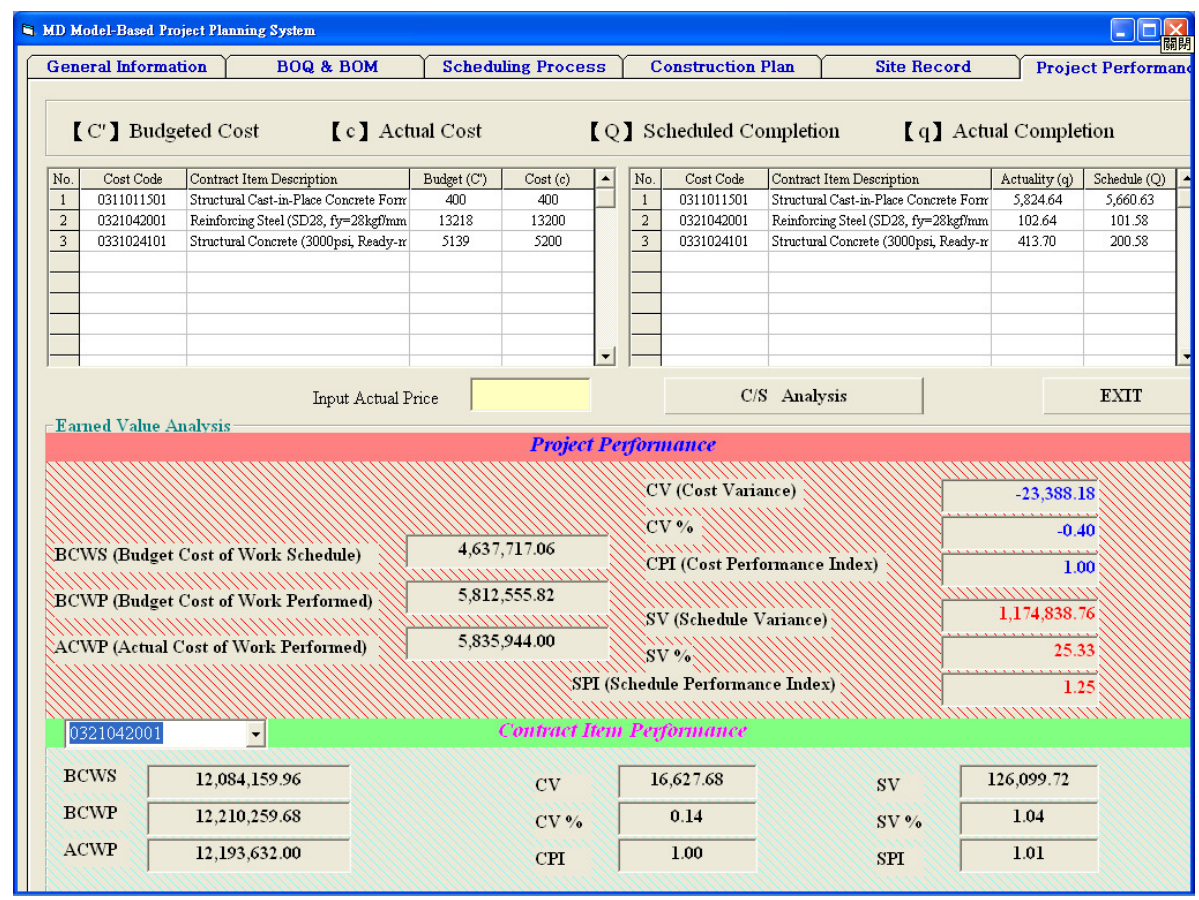

Figure 8. The "Project Performance" section in MD-PPS

\section{REFERENCES}

[1] Waly, A. F., and Thabet, W. Y. (2002) A virtual construction environment for preconstruction planning, Automation in Construction, Vol. 12, 139-154.

[2] Kang, L. S., and Paulson, B. C. (1998) Information Management to Integrate Cost and Schedule for Civil Engineering Projects, ASCE Journal of Construction Engineering and Management, Vol. 124, No. 5, 381389.

[3] Jongeling, R., and Olofsson, T. (2007) A method for planning of work-flow by combined use of locationbased scheduling and 4D CAD, Automation in Construction, Vol. 16, 189-198.

[4] Koo, B., and Fischer, M. (2000) Feasibility Study of 4D CAD in Commercial Construction, ASCE Journal of Construction Engineering and Management, Vol. 126, No. 4, 251-260.

[5] Vries, de, B., and Harink, J. (2007) Generation of a construction planning from a 3D CAD model, Automation in Construction, Vol. 16, 13-18.

[6] Feng, C.W. and Chen, Y.J. (2005) Using MD CAD Objects to Integrate Information for Construction Projects, Proceedings of the 1st International
Conference on Construction Engineering and Management, Seoul, Korea, October 16-19, 515 519.

[7] Koo, B., Fischer, M., and Kunz, J. (2007) A formal identification and re-sequencing process for developing sequencing alternatives in CPM schedules, Automation in Construction, Vol. 17, 75-89.

[8] Tanyer, A. M., and Aouad, G. (2005) Moving beyond the fourth dimension with an IFC-based single project database, Automation in Construction, Vol. $14,15-32$.

[9] Chau, K.W., Anson, M., and Zhang, J. P. (2005) 4D dynamic construction management and visualization software: 1. Development, Automation in Construction, Vol. 14, 512-524.

[10] Dawood, N., and Mallasi, Z. (2006) Construction Workspace Planning: Assignment and Analysis Utilizing 4D Visualization Technologies, ComputerAided Civil and Infrastructure Engineering, Vol. 21, 498-513.

[11] GSA, (2006) Building Information Modeling Guide Series: 01 - GSA BIM Guide Overview, The National 3D-4D-BIM Program Office of the Chief Architect Public Buildings Service, (http://www.gsa.gov/bim). 
[12] IAI, The International Alliance for Interoperability International Web site, http://www.iaiinternational.org/index.html, reviewed in 2007.

[13] buildingSMART Norway, (2007) Information Delivery Manual: Guide to Components and DevelopmentMethods,(http://idm.buildingsmart.com)

[14] Fu, C., Aouad, G., and Lee, A., Mashall-Ponting, A., and $\mathrm{Wu}, \mathrm{S}$. (2006) IFC model viewer to support $\mathrm{nD}$ model application, Automation in Construction, Vol. 15, 178-185.

[15] PCC, (2007) The Public Construction Master Codes and the Public Construction Extended Codes, The Public Construction Commission in Taiwan, (http://www.pcc.gov.tw/).

[16] Feng, C. W., and Chen, Y.J. (2006) Using MD CAD Objects to Automatically Generate A ContractDriven Schedule, Proceedings of the 23rd International Symposium on Automation and Robotics in Construction, Tokyo, Japan, October 3-5, 740-745. 